\author{
Gdańsk 2018, Nr. 39 \\ https://doi.org/10.26881/sgg.2018.39.18 \\ Danuta Olszewska \\ Universität Gdańsk, Philologische Fakultät
}

\title{
Syntaktische Varianz in der deutschen Wissenschaftssprache
}

\begin{abstract}
Wissenschaftliche Texte sind u.a. für ihre Tendenz zur syntaktischen Reduktion bekannt. Aus dem allgemeinsprachlichen Repertoire werden nur bestimmte syntaktische Strukturen für die sachliche und prägnante Darstellung von fachlichen Inhalten ausgewählt und mit hoher Frequenz verwendet. Der Katalog domänentypischer grammatischer Formen umfasst in erster Linie Passivkonstruktionen und Passivumschreibungen, Aussagesätze, Präsens sowie Indikativ. Um das syntaktische Profil wissenschaftlicher Texte zu vervollständigen, werden im Folgenden auch andere Formen präsentiert, die in wissenschaftlichen Texten Anwendung finden und die von einer syntaktischen Varianz dieser Texte sprechen lassen.
\end{abstract}

Schlüsselwörter: Fachtext, Textorganisation, Metaebene, Mediostrukturen

Syntactic Variance in German Scientific Texts. - Scientific texts are noted, inter alia, for a tendency towards grammatical reduction, which consists in the fact that from the general-language repertoire certain syntactic structures are selected and applied that are essential for the objective and concise presentation of scientific content. The catalogue of typical grammatical choices includes, above all, the passive voice and other impersonal constructions, indicative sentences, present tense, and the indicative mood. With the aim of supplementing this simplified syntactic description of scientific texts, this article also demonstrates other grammatical forms that are applied in scientific texts and that make it possible to refer to the grammatical variance of these texts.

Keywords: scientific text, text organization, meta-text, medium-structures

\section{Ist der Stil der Fachtexte varianzarm?}

Schriftbasierte Fachkommunikation hat - wie bekannt - ihre lexikalischen und stilistisch-grammatischen Eigentümlichkeiten. In beiden Bereichen, d. h. im lexikalischen und im grammatischen, genauer gesagt: syntaktischen Bereich, lassen sich zwei gegensätzliche Tendenzen beobachten. Während die lexikalische Ebene der Fachtexte von einer Expansivität, d. h. von einem systematischen Ausbau des Wortschatzes, gekennzeichnet ist, verhält sich ihre syntaktische Ebene im Vergleich zur Allgemeinsprache „eher reduktiv“ in dem Sinne, dass aus ihrem Repertoire nur bestimmte syntaktische Strukturen ausgewählt und mit deutlich höherer Frequenz verwendet werden (Weinrich 1989: 132). Diese Strukturen resultieren aus zwei zentralen stilistischen Maximen, denen Fachtexte generell folgen. Zum einen geht es um sprachliche Prägnanz. Sie ist in der Fachliteratur auch unter solchen Bezeichnungen bekannt wie „Knappheit des Ausdrucks“, „Textkondensation“, „Textkomprimierung“ oder 
„Textverdichtung“ (s. dazu: Kretzenbacher 1991 sowie Fijas 1998). Gemeint ist bei allen diesen Termini das Streben von Textautoren danach, komplexe fachliche Inhalte sprachökonomisch darzustellen. Dem Prinzip der sprachlichen Prägnanz kommen zahlreiche Strukturen aus dem lexikalischen und syntaktischen Bereich entgegen. Im lexikalischen Bereich haben vor allem Nominalisierungen, Univerbierungen, Komposita sowie Abbreviaturen eine textkürzende Wirkung. Auf der Satzebene garantieren ebenfalls mehrere Strukturen eine prägnante Ausdrucksweise, und zwar die Verwendung von Passiv und Passivumschreibungen, satzwertige Infinitiv- und Partizipialkonstruktionen, erweiterte Attribute, Attributketten, Gerundialkonstruktionen sowie Ellipsen (Kretzenbacher 1991, Oksaar 1998).

Ein zweites fachtexttypisches Stilprinzip, das bestimmte syntaktische Strukturen realisieren lassen, bildet die Neutralität. Man kann sie mit solchen Bezeichnungen wie „Sachlichkeit“, „Objektivität“ oder auch „Anonymität“ synonym verwenden. Es geht in Fachtexten darum, die an einen Autor gebundene Subjektivität zu eliminieren und mögliche Allgemeingültigkeit des vermittelten Fachwissens und damit seine Objektivität zu verstärken. Der Realisierung dieser Maxime dienen Konstruktionen, die von den Gesprächsrollen Sender und Empfänger abstrahieren lassen. In erster Linie sind hier deagentivierte Passivsätze und Passivkonkurrenzformen zu nennen, die als grundlegendes Mittel der Objektivierung gelten. Durch Deagentivierung der Verfasserreferenz und Anonymisierung vermittelter Sachverhalte werden lediglich fachliche Informationen fokussiert, so dass der Eindruck eines vom Subjekt (Autor) unabhängigen, objektiven Wissens entsteht. Der Rezipient bekommt damit einen maximal transparenten Text in die Hand (Kretzenbacher 1991).

Im Zusammenhang mit den genannten Stilmaximen, die man auch als fachtexttypische Strategien betrachten kann (Strategie der Textkondensation und Strategie der Objektivierung), spricht Kretzenbacher in Anlehnung an Weinrich und andere Fachtextlinguisten von einer „Varianzreduktion im syntaktischen Bereich“ und von einem „,varianzarmen Stil“ (ebd.: 132). Die syntaktischen Strukturen in Fachtexten zeichnen sich nämlich durch eine negative Quantität aus, die darin besteht, dass die Syntax in der schriftlichen Fachkommunikation auf bestimmte Strukturen reduziert sei. Nominalkonstruktionen, Passivformen, Aussagesätze, Indikativ oder Präsens gehören zu den dominanten und damit fachtexttypischen Mitteln. Solche Formen dagegen wie Konjunktiv, Futur, Frage- oder Aufforderungssätze sowie Bezüge auf die Gesprächsrollen Sender und Empfänger sind nach Kretzenbacher für wissenschaftliche Texte „ungewöhnlich“; sie haben „eine geringe Frequenz“, dafür aber „einen hohen Aufmerksamkeitswert“" d. h. sie bilden ein stilistisches Phänomen (ebd.).

Ohne Unterschiede in der Frequenz der genannten syntaktischen Strukturen in Fachtexten und in der Gemeinsprache in Frage zu stellen, soll im Folgenden ein Spektrum syntaktischer Variabilität in wissenschaftlichen Texten präsentiert werden. Die hier analysierten Formen zählen nicht zu den Formen mit „geringer Frequenz“. Als mehrfach in Analysen erfasste Beispiele können sie ebenfalls als fachtexttypische syntaktische Mittel angesehen werden. Sie tragen zu einer großen stilistischen Varianz bei, die gegenwärtig in Texten beobachtbar ist. Sie bieten den Autoren die Möglichkeit, mit ihren Fachtexten flexibel umzugehen. 


\section{Differenzen bei der Textorganisation}

Ein varianzreicher Stil lässt sich auf der Metaebene wissenschaftlicher Texte beobachten. Auf dieser Ebene kumulieren sich alle explizit realisierten Handlungen, die der Autor zu textkompositorischen und gleichzeitig zu interaktiven Zwecken ausführt. Man kann sie insgesamt als textorganisierende Handlungen betrachten. Ihre Exponenten zählen zu der sog. Allgemeinen Wissenschaftssprache ${ }^{1}$ und sind sowohl für natur- als auch für geisteswissenschaftliche Texte charakteristisch. Sie finden sich genauso in schriftlichen (z.B. Monographien, wissenschaftliche Artikel) wie in mündlichen (z.B. Vorlesungen, Vorträge) Textsorten. Offensichtlich erscheinen textorganisierende Mittel mit unterschiedlicher Frequenz und in verschiedener stilistischer Form. In naturwissenschaftlichen Monographien, die sich durch einen strengen thematisch-funktional motivierten Aufbau auszeichnen, kommen bestimmte Arten von metatextuellen, textorganisierenden Mitteln, wie z.B. Themaankündigungen als Gliederungssignale, viel seltener als in geisteswissenschaftlichen Monographien vor. Auch im Bereich geisteswissenschaftlicher Texte (Monographien und Wissenschaftliche Artikel) bestehen Differenzen zwischen den einzelnen Textexemplaren, einerseits im Hinblick auf die Häufigkeit textorganisierender Äußerungen, andererseits im Hinblick auf ihre stilistische Form. Autoren wissenschaftlicher Texte kommt viel Freiheit zu, nicht nur bei der Wahl von Forschungsthemen, sondern auch bei der Textgestaltung, auch bei der Gestaltung der Metaebene. Die einen verdeutlichen ihre Textbildungsschritte regelmäßig und wollen dem Leser eine möglichst optimale Orientierung im Textraum und Einsicht in ihre Intentionen gewährleisten. Die anderen gehen mit Signalen der Textorganisation sparsam(er) um, was nicht bedeutet, dass ihre Texte schlechter organisiert sind. Die Anzahl von textorganisierenden Mitteln ist nicht unbedingt ein Kriterium für die Qualität eines wissenschaftlichen Textes. Auch die Form dieser Mittel ist es nicht. Bei der Realisierung von textorganisierenden Handlungen richten sich manche nach der Konvention eines unpersönlichen Stils und verwenden in schriftlichen Texten (Monographien und wissenschaftliche Artikel) konsequent eine distanzsprachliche Diktion. Andere wiederum scheuen sich nicht, das selbstbezogene Pronomen ich zu gebrauchen, sei es systematisch im ganzen Text, sei es nur an bestimmten Textstellen oder bei bestimmten Handlungen (mehr dazu Olszewska 2018a). Statistische Untersuchungen zur Erfassung stilistischer Tendenzen in diesem Bereich sind natürlich sinnvoll und wichtig, aber ihrer Aussagekraft sind Grenzen gesetzt. Denn jedem ausgewählten Korpus kann man ein Korpus gegenüberstellen, das auf andere Tendenzen hinweisen kann. Auch das umfangreichste Korpus führt letzten Endes zu der Konklusion, dass im Hinblick auf die Textorganisation Differenzen zwischen den Texten bestehen.

Dem folgenden Beitrag liegen keine detaillierten statistischen Untersuchungen zugrunde. Sein Ziel ist es, das syntaktische Potenzial von Fachtexten auf ihrer Metaebene zu zeigen,

\footnotetext{
1 Der Begriff „Allgemeine Wissenschaftssprache“, anders gesagt „Alltägliche Wissenschaftssprache“ stammt von Konrad Ehlich. Der Autor zählt dazu „Sprachliche Formen, die einerseits an der alltäglichen Sprache unmittelbar teilhaben, die andererseits einen elementaren Bestand von Ausdrucksmitteln für die Wissenschaftskommunikation zur Verfügung halten, ohne den diese nicht vorstellbar wäre“ (Ehlich 2006: 25).
} 
ein Potential aus dem die Autoren heutzutage schöpfen und eine weitgehende individuelle Varianz aufbauen. ${ }^{2}$

Als Materialbasis dienen geisteswissenschaftliche Textsorten: Monographien und Artikel aus den Bereichen Linguistik, Literaturwissenschaft und Sozialwissenschaften. Das Verzeichnis von Monographien befindet sich in Olszewska (2007) und in Olszewska (2018a).

\section{Varianzreichtum bei der Textorganisation}

Bei der Realisierung von gleichen bzw. ähnlichen Intentionen konkurrieren auf der Metaebene wissenschaftlicher Texte mehrere grammatische Strukturen. Ihre Konkurrenz ergibt eine weitgehende grammatische Synonymie. Die stärkste Konkurrenz lässt sich wohl zwischen Aktiv- und Passivsätzen beobachten:

\section{a) Passiv vs. Aktiv}

Zweifellos sind wissenschaftliche Texte wie Monographien und wissenschaftliche Artikel passivfreundliche Texte. Auf einen hohen Anteil von Passivkonstruktionen und Passivkonkurrenzformen haben viele Linguisten hingewiesen, seitdem sich die Fachsprachenforschung in den 70er Jahren zu entwickeln begann (z.B. Beneš 1981, Bungarten 1981, Panther 1981, Hoffmann 1984, Baumann 1987, Kretzenbacher 1991). Passiv- und passivähnliche, in diesem Sinne unpersönliche Formen ermöglichen eine Deagentivierung und damit die Eliminierung des Autorsubjekts aus der Textoberfläche. Diese sprachliche Strategie soll einerseits einen sachlichen, objektivierten Stil, andererseits eine Textkondensation gewährleisten, die durch die Valenzreduktion des Verbs bedingt ist. Obwohl die unpersönlichen Konstruktionen und die damit verbundene Vorherrschaft der 3. Person ein hochfrequentes fachtexttypisches Phänomen darstellen, sind persönliche Formen im Aktiv, d. h. Äußerungen in der 1. Person Sg, gegenwärtig - sei es in deutschen, sei es in polnischen Wissenschaftstexten - keine Seltenheit. Bei textorganisierenden Handlungen bilden sie heutzutage relativ häufige Alternativen und grammatische Synonyme für Passivformen. Die ungeschriebene Regel: Ein Wissenschaftler sagt nicht, ich", die von Weinrich (1989: 132) als ein „Ich-Verbot" und die von Kretzenbacher (1994: 27 f.) als ein „Ich-Tabu“ formuliert wurde, findet in der heutigen Schreibpraxis keine Bestätigung. Die konventionelle Musterhaftigkeit, verstanden als 'Sprache der Distanz' und sachlicher, entpersönlichter Stil, scheint für viele Autoren zu eng zu sein. Zu Wort kommt mehr individuelle Varianz, die eine Abkehr von der schriftsprachlichen Konvention und eine Hinwendung zur konzeptionellen Mündlichkeit, anders gesagt zur 'Sprache der Nähe',

2 Der Begriff ,individuelle Varianz” stammt von Petkova-Kessanlis (2009), den die Autorin neben den Begriffen „musterbezogene Varianz“ und „originelle Varianz“ verwendet. Bei der „musterbezogenen Varianz“ geht es um Textrealisierungen, die einer entsprechenden Konvention folgen und im Textmuster enthalten sind. Die konventionelle, musterbezogene Varianz wird mit ihrem Potenzial verfügbarer Mittel in konkreten Textexemplaren individuell umgesetzt und in dem Sinne kann man von einer individuellen Varianz bei der Textrealisierung sprechen (Petkova-Kessanlis 2009: 3). 
bedeutet. Es ist schwierig, einen allgemeingültigen Grund für den Gebrauch des Pronomens ich anzugeben, aber man kann annehmen, dass als Hauptgrund dafür das Bedürfnis nach Natürlichkeit, Direktheit und Authentizität ist. Das Begriffspaar „Distanz - Nähe“, das Koch und Oesterreicher (1986) bei der Beschreibung des Distanz-Nähe-Kontinuums eingeführt haben, kann als eine evaluative Polarisierung verstanden werden. „Nähe konnotiert positiv, ruft Werte wie Gemeinsamkeit, [...] Interaktion auf, ,Distanz' konnotiert negativ, ruft Werte wie Fremdheit, Bürokratie, Behörde etc. auf " - bemerkt Knobloch bei der Diskussion um das Nähe-Distanz-Konzept über dreißig Jahre später und stellt die Frage: „Warum sollte Schriftlichkeit weniger natürlich sein als Mündlichkeit?“" Knobloch 2017: 76).

Die durch das Pronomen ich markierte und in schriftlichen Texten inszenierte Mündlichkeit begleitet in gegenwärtigen deutschen Wissenschaftstexten viele textorganisierende Handlungen. Sie ist zunächst bei Themaankündigungen und Themapräzisierungen beobachtbar, die als Gliederungssignale in geisteswissenschaftlichen Texten häufig verwendet werden, z.B.:

\section{Passiv}

Im Folgenden werden... näher erläutert.

Zum Schluss soll noch auf ... eingegangen werden.

Dabei wird das Thema auf ... eingeengt.

Damit wird die Diskussion ... abgeschlossen. Im nächsten Kapitel wird ... dargestellt.

\section{Aktiv}

Im Folgenden möchte ich ... näher erläutern. Zum Schluss will ich noch auf... kurz eingehen. Dabei beschränke ich mich auf ...

Damit schließe ich die Diskussion ... ab. Im nächsten Kapitel wende ich mich ... zu.

Ich-Formulierungen finden sich auch bei Themaverschiebungen, die darüber informieren, dass ein angedeutetes Teilthema an einer späteren Textstelle ausführlich(er) behandelt wird. Damit sichert der Autor dem Leser eine Orientierung im thematisch geordneten Aufbau des Textes ab:

Passiv

Darauf wird in Kap. $x$ näher eingegangen.

Diese Frage wird in $\mathrm{x}$ ausführlich behandelt.
Aktiv

Darauf gehe ich in Kap. $x$ näher ein.

Diese Frage bespreche ich in $\mathrm{x}$ ausführlicher.

Die Autoren verwenden das Pronomen ich bei der Ankündigung zusammenfassender Gedanken, die einen größeren Propositionskomplex, z.B. ein Hauptkapitel abschließen:

Passivumschreibung

Zusammenfassend ist festzuhalten, dass ... Resümierend lässt sich sagen, dass ...
Aktiv

Ich fasse zusammen: ... Ich rekapituliere: ...

Relativ häufig erscheint das Pronomen ich in performativen Ausdrücken bei begriffsbildenden Prozeduren, in denen der Autor seine Rolle als eines Forschers verdeutlicht und den Leser über seine individuellen, methodologisch-begrifflichen Entscheidungen informiert. Durch einen expliziten Bezug auf sich selbst wollen die Autoren verdeutlichen, dass sie selbstständig 
handeln, autonom sind und die Verantwortung für ihre Entscheidungen tragen (mehr dazu Olszewska 2018a). Beispiele:

Passiv

Unter x soll im Folgenden ... verstanden werden. Als $\mathrm{x}$ werden im Folgenden ... bezeichnet.

Im Folgenden wird davon ausgegangen, dass ...

Es wird hier angenommen, dass ...

Aus der Analyse wurden ... ausgeklammert.

Unterschieden werden hier folgende ...
Aktiv

Unter $\mathrm{x}$ verstehe ich hier ... Ich bezeichne sie als ...

Im Folgenden gehe ich davon aus, dass ... Dabei nehme ich an, dass ...

Bei der Analyse berücksichtige ich ... nicht.

Ich unterscheide folgende ...

Aus ähnlichen Gründen, d.h. um ihre Autonomie und Verantwortung für getroffene Entscheidungen zu verdeutlichen, neigen die Autoren zum Gebrauch von ich bei Handlungen, in denen sie ihre eigene Stellungnahme manifestieren wollen:

Passiv

Im Folgenden wird (nicht) behauptet, dass ...

Dagegen kann eingewendet werden, dass ...

Es kann hier vermutet werden, dass ...
Aktiv

Ich behaupte (nicht), dass ...

Dagegen möchte ich einwenden, dass ... Anders als x möchte ich festhalten, dass ... Ich stimme $\mathrm{x} z u$, wenn er feststellt, dass ... Ich schließe mich $\mathrm{x}$ an, die ... Ich vermute, dass ... / Es scheint mir, dass ...

Die deagentivierten Passivsätze (links) repräsentieren die konzeptionelle Schriftlichkeit und die 'Sprache der Distanz'. Ihre persönlichen Konkurrenzformen (rechts) stehen für die 'Sprache der Nähe’ und treten in erster Linie in Vorlesungen bzw. Vorträgen auf. Die Autoren übertragen sie aber auch auf schriftliche Texte, inszenieren damit die konzeptionelle Mündlichkeit und erwecken beim Leser den Eindruck einer fiktiven Gesprächssituation (mehr dazu Olszewska 2018 b).

\section{b) Aussagesatz vs. Fragesatz vs. Aufforderungssatz}

Die führende Position der Aussagesätze in wissenschaftlichen Texten ist nicht zu leugnen. Frage- sowie Aufforderungssätze stellen jedoch in diesem Kommunikationsbereich keine Randerscheinung dar. Fragesätze bilden einen festen Bestandteil wissenschaftlicher Texte als disputierender und problemlösender Texte. Fragen zu stellen, bedeutet das Problematisieren, das eine Grundlage für jeden Forschungsprozess und damit für wissenschaftliche Texte ist. In einer Vorlesung oder einem Vortrag sind Fragen natürliche Formen einer unmittelbaren Interaktion. In schriftlichen Texten, die medial gesehen monologische Texte sind, kann der Autor als Fragender keine unmittelbare Reaktion der Leser erwarten. Trotzdem werden beim Schreiben Fragen an potenzielle, unbekannte Leser mit unbekanntem Wissensstand gestellt. Zum einen geht es hier um indirekte Fragesätze, die als Nebensätze (Komplementsätze) einem Hauptsatz angeschlossen werden. Bei der Formulierung von Zielangaben stellt der Autor eine problematisierende Frage, auf die er im Rahmen seiner Untersuchung eine Antwort 
suchen wird. Man kann diese Fragen auch als Antizipationen von der Seite interessierter Leser verstehen. Zu den Routineformeln können z.B. folgende Formulierungsmuster gezählt werden, die in einer Einleitung oder am Anfang eines Kapitels beim Präzisieren des Untersuchungsziels verwendet werden:

Im Folgenden wird der Frage nachgegangen, wie / inwieweit / welche / u. ä.

Im Folgenden soll die Frage beantwortet werden, wie / warum / woraus / u. ä.

In diesem Kapitel wird untersucht, ob / inwiefern / u. ä.

Es geht dabei um die Frage, ob / wie / u. ä.

Es drängt sich hier die Frage auf, ob und inwieweit / wodurch u. ä.

Es stellt sich in diesem Kontext die Frage, was / u. ä.

$\mathrm{Zu}$ fragen ist nun, ob / inwiefern / u. ä.

Zum anderen begegnet man nicht selten direkten Ergänzungs- oder Entscheidungsfragen, die in Argumentationsstrukturen eingesetzt werden, eine rhetorische Perspektive schaffen und die Leser auf die Lösung „kleinerer“ Teilprobleme vorbereiten. Beim Übergang zu einem neuen Teilaspekt kann man sich also solche Fragen als Themenwechselsignale vorstellen, wie:

Wie ist das zu erklären?

Woran ist die Erscheinung erkennbar?

Was heißt aber $\mathrm{x}$ ?

Welche Faktoren können dabei noch eine Rolle spielen?

Tragen dazu nur ... bei?

Insgesamt verleihen Fragen (sowohl indirekte Fragesätze als auch direkte Fragen) wissenschaftlichen Texten einen interaktiven Charakter: Sie beziehen den Leser in die Untersuchungssituation mit ein, verstärken die Performativität dieser Texte, d. h. den Eindruck, als würde sich die Untersuchung hic et nunc abspielen. Damit nähern sie einen Text dem Pol der konzeptionellen Mündlichkeit an. Der Autor schreibt, als ob er einen Vortrag halten und gemeinsam mit dem Leser nach einer Lösung strittiger Fragen streben würde (vgl. dazu Olszewska 2018 b).

Der Interaktionsgrad eines wissenschaftlichen Textes wird auch durch den Einsatz von Aufforderungssätzen erhöht, in denen die Personen $d u / i h r$ noch stärker als in Fragesätzen involviert sind. Aufforderungssätze mit dem leserinklusiven wir sind generell expressive Mittel der Aufmerksamkeitssteuerung, die an bestimmten Textstellen zu einem stilistischen Zweck verwendet werden, d. h. um eine rhetorische Perspektive zu schaffen, den Stil lebendiger zu machen und auf den Leser stärker einzuwirken. Die Autoren machen von solchen Formen beim Themenwechsel innerhalb der Mediostrukturen Gebrauch, wenn sie die Aufmerksamkeit des Lesers auf einen neuen thematischen Aspekt lenken wollen. ${ }^{3}$ In diesen Fällen sind sie also Mittel zur Umfokussierung. Anders gesagt, sind sie Themenwechselsignale. Als Konkurrenzformen und grammatische Synonyme können hier Sätze im Aussagemodus (links) genannt werden, die ebenfalls einen Übergang zu einem neuen Schritt bei der

\footnotetext{
3 „Mediostrukturen“ liegen nach Engel zwischen der Makrostruktur eines Textes (Grobstruktur) und der Mikrostruktur als einer Struktur der einzelnen Äußerung (Engel 1988: 34 und 105). Mediostrukturen, die unterschiedlichen Komplexitätsgrad haben, kann man mit einer Mesoebene gleichsetzen, d.h. mit der Ebene der Absätze.
} 
Themenbehandlung markieren, allerdings keinen stilistischen Mehrwert an Expressivität und Textdynamik aufweisen:

\section{Aussagesatz}

Ich wende mich nun ... zu.

Nun will ich zu ... übergehen.

Ich komme jetzt auf ... zurück.
Aufforderungssatz

Wenden wir uns jetzt ... zu

Gehen wir nun zu ... über.

Kommen wir jetzt auf ... zurück.

Sätze im Aufforderungsmodus können auch als richtige Kooperationsangebote eingesetzt werden, die der Autor an den Leser richtet, um ihn zu einer gemeinsamen mentalen Aktivität zu bewegen und ihn für die Lösung des behandelten Problems zu gewinnen. Solche expliziten Kooperationsangebote mit einem Analyse-Anreiz lassen sich an folgenden Textstellen beobachten:

- beim Themenwechsel innerhalb der Mediostrukturen, wenn der Autor ein besonderes Problem näher analysieren will. Als Routineausdrücke können folgende Formulierungen gelten:

Betrachten wir nun ... etwas näher.

Schauen wir uns diese ... etwas näher an.

Vergleichen wir ...

Werfen wir jetzt unseren Blick auf ...

- bei der Beispielgebung, wenn der Autor ein besonderes Beispiel präsentieren und es später erläutern will, z.B.:

Nehmen wir ein folgendes Beispiel;

Betrachten wir ein anderes Beispiel näher;

Stellen wir uns die folgende Situation vor;

Vergleichen wir folgende Beispiele; u. ä.

Das gemeinsame Betrachten eines Teilproblems oder spezieller Beispiele berechtigt den Autor wiederum dazu, den Leser zu einem gemeinsamen Schlussfolgern oder Resümieren „einzuladen“. Auch bei diesen wichtigen Handlungen wollen die Autoren mit den Lesern „kooperieren“ und abschließende Gedanken als gemeinsames Gut herausarbeiten. Dies können die folgenden, rechts stehenden Formen mit dem „kooperativen“ wir bestätigen. Nicht selten konkurrieren sie mit den links angegebenen Alternativen:

\section{Aussagesätze}

Ich fasse zusammen: ...

Resümierend lässt sich sagen, dass ...

Zusammenfassend ist festzuhalten, dass ...

Aus dem Gesagten ergibt sich Folgendes: ...

Aus ... kann die Schlussfolgerung gezogen

werden, dass ...
Aufforderungssatz / Fragesatz

Fassen wir zusammen: ..

Rekapitulieren wir: ...

Halten wir fest: ...

Was ergibt sich aus dem Gesagten?

Welche Schlussfolgerungen lassen

sich ... ziehen? 
c) Indikativ vs. Konjunktiv I

Ähnlich wie in der Allgemeinsprache ist der Indikativ als Wirklichkeitsmodus eine dominante Moduskategorie in wissenschaftlichen Schrifttexten. Er dient zur sachlichen Darstellung fachlicher Inhalte. Anders verhält es sich mit dem Konjunktiv I. Während diese Kategorie in der Allgemeinsprache eine geringe Rolle spielt, ist sie in wissenschaftlichen Fachtexten keine Seltenheit. Gemeint ist dabei nicht so sehr der Konjunktiv I in seiner Hauptfunktion, d.h. als Konjunktiv zur Signalisierung indirekter Rede (Duden Bd. 4, 2006: 529; Helbig / Buscha 2001: 174), die bei Bezügen auf fremde Texte und Meinungen anderer Autoren häufig verwendet wird, als viel mehr der Konjunktiv I in seiner Nebenfunktion: als sog. „,adhortativer Konjunktiv“" (Engel 1988: 419). Mit dem „adhortativen Konjunktiv“ ist ein auffordernder Konjunktiv gemeint, der einen Sachverhalt als „erwünscht“ oder als „postuliert“ bezeichnet (Engel 1999: 607). Genauer gesagt geht es um den Konjunktiv I von sein in Verbindung mit dem Part. II passivfähiger Verben. Diese Form (sei + Part. II) konkurriert in wissenschaftlichen Texten mit dem Indikativ bei textorganisierenden Handlungen auf der Metaebene. Damit trägt der Konjunktiv I zur syntaktischen Varianz und zur grammatischen Synonymie bei. Die Substitution von Indikativ und Konjunktiv I in Form von sei + Part. II ist für solche Äußerungen aktuell, mit deren Hilfe der Autor verdeutlicht, dass er bestimmte Inhalte vermitteln will, die ihm für die Entwicklung eines Themas als relevant erscheinen. Semantisch-pragmatisch steht die Modalität des Wunsches im Falle von sei + Part. II der Modalität der Notwendigkeit nah. Daher kann diese Form in wissenschaftlichen Texten als Konkurrenzform für die passivische Konstruktion sein $+z u+$ Infinitiv sowie für das Passiv mit sollen angesehen werden. Allerdings klingen die passivischen Indikativformen „etwas kategorischer als die im Konjunktiv I“ (Engel 1999: 608), „der Bedeutungsunterschied bleibt aber gering" (Engel 1988: 420).

Die konjunktivische Form sei+ Part. II und ihre indikativischen Alternativen sind charakteristisch für mehrere textorganisierende Handlungen sowie für unterschiedliche Kontexte, die den Vermittlungsprozess und die einzelnen Textbildungsphasen abbilden. Generell erfüllen sie eine textstrukturierende Funktion. Dabei geht es um drei Teilfunktionen, die mit drei textorganisierenden Handlungen zusammenhängen, und zwar: GLIEDERN, VERKNüPfEN und Hierarchisieren. Beim Gliedern kommen drei weitere textorganisierende Handlungen in Frage, nämlich: Initiieren, Wechseln, und Schliessen. Der Vollzug dieser Handlungen ergibt einen übersichtlichen und damit verständlichen Text, so dass man sagen kann, dass die Metaformulierungen eine textstrukturierende, anders gesagt eine textkompositorische, und gleichzeitig auch eine interaktive Funktion erfüllen.

Die genannten textorganisierenden Handlungen sind für vier große Gruppen von Metaformulierungen charakteristisch, sei es für diejenigen im Indikativ, sei es für diejenigen im Konjunktiv I. Eine Gruppe bilden die Thematisierungen, mit deren Hilfe der Autor ein Teilthema im Sinne eines Problems ankündigt, das im Rahmen eines Themas beispielsweise in einem Kapitel behandelt wird. Wissenschaftliche Texte sind in der Regel inhaltlich recht komplex und werden nicht nur mit Hilfe von Überschriften, sondern auch mittels Thematisierungen und Teilthematisierungen in kleinere thematische Komplexe gegliedert. Ähnlich verhält es sich mit Verweisen, die dazu dienen, fremde Texte und Meinungen 
anderer Autoren in den Text einzubinden sowie durch Exemplifizierungssignale Beispiele in den Text zu integrieren. Auch diese Textelemente müssen - wie die einzelnen thematischen Aspekte - auf eine geordnete Art und Weise präsentiert werden. Die vierte Gruppe bilden metatextuell markierte Assertionen, die für Einzelpropositionen stehen. Assertionen haben die Aufgabe, Wissen zu vermitteln und bilden in wissenschaftlichen Texten ein konstitutives Handlungsmuster. Aus der assertorischen Oberfläche eines Textes „,ragen“ nun Assertionen „heraus“, die durch einen metatextuellen Matrixsatz markiert sind. Durch diesen Matrixsatz verdeutlicht der Autor sein Assertieren und „verrät" damit Informationen z.B. zur Textstrukturierung oder zu seiner Einstellung gegenüber der im nachfolgenden dass-Satz vermittelten wissenschaftlichen Proposition. Die vier genannten Gruppen von Metaformulierungen resultieren aus dem Bedürfnis des Autors, die einzelnen Textkomponenten wie Teilthemen, Verweise auf andere Texte, Beispiele sowie bestimmte Einzelpropositionen zu Übersichtlichkeitszwecken voneinander abzugrenzen und so den Leser auf die Rezeption immer neuer Inhalte vorzubereiten.

Im Rahmen der Gliederungsfunktion werden die Metaformulierungen zunächst als Initialsignale eingesetzt. Man kann sie auch mit Engel (1988: 105) als Vorschaltungen bezeichnen. Sie nehmen eine exponierte, $d$. h. eine absatzinitiale, Position ein und signalisieren: ein erstes Teilthema, einen ersten Verweis, ein erstes Beispiel oder eine erste spezielle Proposition. Ihre typischen lexikalischen Komponenten sind solche temporalen Sequenzausdrücke wie zunächst, zu Beginn, von Anfang an, bevor-Satz, vor-Präpositionalphrase, vorausschickend, bereits hier $\mathrm{u}$. ä. Im Falle von Thematisierungen, Exemplifizierungssignalen und metatextuell markierten Assertionen verdeutlichen sie Folgendes: Bevor der Autor zum eigentlichen Argumentationsstrang übergeht, hält er für notwendig, ein bestimmtes Problem, ein bestimmtes Beispiel oder eine spezielle Proposition in den Text einzuführen. Als „spezielle Propositionen“ gelten meistens präzisierend-einschränkende Hinweise methodologischer Art, die vor allem den Interessen des Autors und seinem Selbstschutz dienen, gleichzeitig aber auch das Verständnis sichern sollen. Im Falle von Verweisen geht es um einen ersten Bezug auf einen anderen Autor. Die links und rechts angegebenen, grammatisch differenzierten Metaformulierungen repräsentieren die oben genannten Gruppen von Metaformulierungen und gelten als grammatische Äquivalente:

Indikativ

Teilthematisierungen:

Zunächst soll auf Folgendes hingewiesen werden:...

Vor der Behandlung der ... sind einige Begriffe zu erläutern.

Verweise:

Zunächst ist auf $\mathrm{x}$ zu verweisen, der ... Als erste soll die Definition von x zitiert werden:
Konjunktiv I

Zunächst sei auf Folgendes hingewiesen: ...

Zunächst seien einige Begriffe erklärt.

Zunächst sei auf $\mathrm{x}$ verwiesen, der ... Als erste sei die Definition von x zitiert: ... 
Exemplifizierungssignale:

Zu Beginn sind folgende Beispiele anzuführen: ...

Bevor..., seien folgende Beispiele angeführt: ...

Ich möchte vom folgenden Beispiel ausgehen:

Zunächst sei ein folgendes Beispiel präsentiert: ..

Metatextuelle Einleitungsformeln, die spezielle Propositionen einführen:

Bevor ... näher behandelt werden,

ist zu betonen, dass...

Vorausschickend ist zu bemerken, dass ...

Um Missverständnissen vorzubeugen, ist bereits darauf hinzuweisen, dass ...
Bevor..., sei hervorgehoben, dass ...

Es sei von Anfang an betont, dass ...

Um Missverständnisse auszuklammern, sei hier darauf hingewiesen, dass ...

Diese grammatischen Formen werden auch als Wechselsignale verwendet. In ihrer propositionalen Struktur erscheinen diesmal Sequenzausdrücke, die auf eine weitere Position der jeweiligen Textkomponente (Teilthema, Verweis, Beispiel oder neuer Sachverhalt) innerhalb einer Ausführung hinweisen, wie z.B. noch, auch, ein weiteres Problem, eine weitere Frage, eine andere Frage, ferner, darüber hinaus, außerdem, des Weiteren u. ä. Als zusätzliche lexikalische Füllungen können auch konnektive Ausdrücke erscheinen, die die neuen Sachverhalte an den aktuellen Kontext explizit binden, wie z.B. in diesem Zusammenhang, dabei, hier, an dieser Stelle. Auch diese Metaformulierungen gelten als Routineformeln; sie stehen absatzinitial und verdeutlichen einen Übergang zu einem neuen Argumentationsschritt:

Indikativ

Teilthematisierungen:

Noch eine ... soll hier erwähnt werden. ...

In diesem Zusammenhang soll auch auf die ... hingewiesen werden....

An dieser Stelle ist noch die Frage ... anzusprechen. Noch eine Frage sei an dieser Stelle angedeutet.
Noch eine ... sei hier erwähnt. ...

In diesem Zusammenhang sei auch auf die ... hingewiesen. ...
Ferner soll auf $\mathrm{x}$ hingewiesen werden, der / die ...

An dieser Stelle ist auch $\mathrm{x}$ zu nennen, dessen Konzept ...

$\mathrm{Zu}$ verweisen ist hier auch auf $\mathrm{x}, \mathrm{der} / \mathrm{die}$...

Exemplifizierungssignale:

Als nächstes Beispiel soll der folgende Satz zitiert werden.

\section{Verweise:}

Weiterhin sei auf $\mathrm{x}$ verwiesen, der / die ...

In diesem Kontext sei auch $\mathrm{x}$ genannt, der ...

Verwiesen sei hier auch auf $\mathrm{x}$, der / die ...

Als nächstes Beispiel sei folgender Satz zitiert: 
Metatextuelle Matrixsätze, die spezielle Propositionen einführen:

Ferner ist darauf hinzuweisen, dass ... Ferner sei darauf hingewiesen, dass ...

Dabei ist auch hervorzuheben, dass ... Dabei sei auch hervorgehoben, dass ...

Darüber hinaus soll bemerkt werden, dass ...

Des Weiteren sei hier bemerkt, dass ...

Die indikativischen Formen und der Konjunktiv I konkurrieren auch bei der Funktion der Abschlusssignale, die man mit Engel (1988: 105) auch Nachschaltungen nennen kann. Als Indikatoren für diese textstrukturierende Funktion gelten Sequenzausdrücke, die auf die letzte Position einer Textkomponente hinweisen: schließlich, abschließend, zum Schluss, als letzte/r/s, noch. Als zusätzliches lexikalisches Element dieser Metaformulierungen erscheint relativ häufig auch der Selektionsausdruck kurz, der mit den oben genannten Sequenzausdrücken kombiniert wird und den Autor von einer ausführlichen Behandlung eines Problems „,befreit“. Die von den Abschlusssignalen angekündigten Sachverhalte haben nicht selten einen ergänzenden Charakter und können Nebenstrukturen bilden, worauf die hier verwendeten Meta-Prädikate hindeuten. Bei Thematisierungen sind es häufig hinweisen auf etw., andeuten, anschneiden, ansprechen. Bei metatextuellen Einleitungsformeln dagegen wiederholen sich oft anmerken, bemerken, hinzufügen, erwähnen u. ä. Damit können diese Formulierungsmuster auch eine hierarchisierende Funktion erfüllen und Nebenstrukturen von Hauptstrukturen abgrenzen lassen. Zu den typischen Abschlusssignalen können hier die folgenden grammatisch differenzierten Routineformeln gezählt werden:

$$
\text { Indikativ }
$$

Thematisierungen:

Schließlich soll noch kurz auf ...

hingewiesen werden. ...

Abschließend ist kurz auf zweierlei hinzuweisen....

Letztlich soll ... nur kurz angeschnitten werden. ...

Verweise:

Schließlich ist x zu erwähnen. ...

Zum Schluss soll auch auf $\mathrm{x}$ verwiesen werden. ...

Exemplifizierungssignale:

Zum Schluss sind folgende Wörter

anzuführen:...
Konjunktiv I

Schließlich sei hier noch auf ... hingewiesen. ...

Zum Schluss sei noch ... kurz angesprochen....

Abschließend sei noch auf ... hingewiesen....

Schließlich sei x erwähnt. ...

Zum Schluss sei auch auf $\mathrm{x}$ verwiesen. ...

Zum Schluss seien folgende Wörter angeführt: ... 
Metatextuelle Einleitungsformeln, die spezielle Propositionen einführen:

Schließlich ist anzumerken, dass ...

Schließlich soll erwähnt werden, dass ...

Erwähnung verdient schließlich die Tatsache, dass ...

Zum Schluss ist hinzuzufügen, dass ...

Zum Schluss ist zu bemerken, dass ...

Ergänzend ist zu vermerken, dass ...
Schließlich sei angemerkt, dass ...

Zum Schluss sei erwähnt, dass ...

Der Vollständigkeit halber sei erwähnt, dass ...

Abschließend sei hinzugefügt, dass ...

Abschließend sei darauf hingewiesen, dass ...

Ergänzend sei hier angemerkt, dass ...

Die abschließende Funktion der konjunktivischen Form sei + Part. II manifestiert sich deutlich in ihrer Kombination mit einer Dethematisierung oder mit einer Suspendierung als Voräußerungen. Beispiele:

Kombination: Dethematisierung + Abschlusssignal im Konjunktiv I:

Diese Frage kann hier nicht weiter diskutiert werden. Hier sei nur angedeutet, dass ...

Auf weitere ... muss hier verzichtet werden. Ergänzend sei hier lediglich darauf hingewiesen, dass ...

Kombination: Suspendierung + Abschlusssignal im Konjunktiv I:

Dieser Aspekt wird später genauer behandelt. An dieser Stelle sei nur angemerkt, dass ...

Auf diese Frage gehe ich unten näher ein. Vorgreifend sei hier nur darauf hingewiesen, dass ...

Im Rahmen der textstrukturierenden Funktion erfüllen Metaformulierungen, wie oben angedeutet, auch eine verknüpfende Funktion. Die in Segmente gegliederten Textkomponenten, wie Teilthemen, Verweise oder Einzelpropositionen, müssen gleichzeitig miteinander verbunden werden und als eine kohärente Ganzheit erscheinen. Auch hier, d. h. im Bereich der Metaformulierungen mit konnektiver Funktion, lässt sich eine Konkurrenz zwischen dem Indikativ und dem Konjunktiv I in Form von sei +Part. II beobachten. Sie wird im Folgenden am Beispiel von metatextuell markierten Assertionen gezeigt. Als Hauptkomponenten dieser Formulierungsmuster gelten jetzt einerseits thematische Konnektoren wie dabei, hierbei, in diesem Zusammenhang, in diesem Kontext, die eine thematische Nähe zwischen der neu eingeführten Proposition und dem früher Gesagten verdeutlichen. Als äquivalente Formen für diese Ausdrücke können die kontextbezogenen Lokalausdrücke hier und an dieser Stelle angesehen werden, die es ermöglichen, dem vorhandenen, aktuellen Kontext einen neuen thematisch passenden Sachverhalt anzuschließen. Andererseits enthalten diese Metaformulierungen argumentative Konnektoren, d. h. Ausdrücke, die für argumentative Muster typisch sind, wie aber, jedoch, allerdings, immerhin. Metaformulierungen mit ihnen führen eine neue Proposition ein und setzen diese in eine argumentative Relation zur Voräußerung. Weinrich nennt sie Nexusadverbien, womit ihre weniger konnektive Wirkung als viel mehr 
ihre Kohärenzkraft bei der Verbindung von zwei Propositionen verdeutlicht wird (Weinrich 1993: 600). Engel dagegen bezeichnet sie als Textorganisatoren: Sie „verbinden Äußerungen mit dem umgebenden Text; insofern ,organisieren' sie den Text als Ganzes“(1988: 89).

Metaformulierungen, die die genannten konnektiven Ausdrücke enthalten und die eine neue Proposition in den Text einführen, werden an unterschiedlichen Positionen innerhalb eines Argumentationsstrangs situiert. Die metatextuell eingeführte Proposition kann sich auf den weiteren Textverlauf expansiv auswirken, d. h. sie kann die Gesamtargumentation erweitern und so das Thema fortführen lassen. In diesen Fällen nehmen die Metaformulierungen eine strategische absatzinitiale Stellung ein und haben in ihrem Skopus einen ganzen Absatz oder mehrere Absätze, die zur Hauptstruktur des Textes gehören. Als Meta-Prädikate sind in solchen Fällen themastrukturierende Verben typisch, mit deren Hilfe der Autor die Aufmerksamkeit des Lesers stärker steuern und der Argumentation eine bestimmte Richtung geben kann. Hochfrequent sind z.B. die Verben hinweisen aufetw., hervorheben und betonen, die sowohl in indikativischen Formen auftreten als auch mit der konjunktivischen Form sei + Part. II kompatibel sind. Von beiden Formen machen die Autoren Gebrauch, wenn sie die eingeführten Inhalte für notwendig halten:

Indikativ

Dabei soll auf Folgendes hingewiesen werden: ...

In diesem Zusammenhang ist hervorzuheben, dass ...

Dabei ist wichtig zu betonen, dass ...

Dabei soll jedoch darauf hingewiesen werden, dass ...
Konjunktiv I

Dabei sei auf Folgendes hingewiesen. ...

In diesem Zusammenhang sei betont, dass ...

Hierbei sei hervorgehoben, dass ...

Allerdings sei hervorgehoben, dass ...

Die genannten Formulierungsmuster können aber auch eine Nebenstruktur einführen und diese von der obigen Hauptstruktur abgrenzen. Die eingeführten Inhalte sollen das bisher Gesagte ergänzen oder aber einschränken. Für den Autor sind sie wichtig und er „kündigt “ sie mit der Modalität der Notwendigkeit „an“. Über den ergänzenden bzw. einschränkenden Charakter der eingeführten Sachverhalte können Meta-Prädikate oder zusätzliche Elemente Auskunft geben. Typisch sind hier z.B.: anmerken, bemerken, erwähnen oder erinnern an etw. Als Zusätze, die den ergänzenden oder einschränkenden Charakter der eingeführten Proposition verdeutlichen sollen, können die expliziten Signale in Form von ergänzend oder einschränkend erscheinen. Beispiele:

Indikativ

Dabei ist anzumerken, dass ...

Hierbei soll erwähnt werden, dass ...

Allerdings ist dabei zu erwähnen, dass ...

Dabei soll daran erinnert werden, dass ...

\section{Konjunktiv I}

Dabei sei angemerkt, dass ...

Hierbei sei erwähnt, dass ...

Allerdings sei an dieser Stelle erwähnt, dass ...

In diesem Kontext sei daran erinnert, dass ... 
Die genannten konnektiven Metaformulierungen können auch eine neue Proposition in den Text einführen, die die Gesamtargumentation thematisch abrunden und diese abschließen soll. In diesen Fällen stehen die genannten Textroutinen absatzfinal. Insgesamt sind die oben präsentierten Metaformulierungen sowohl grammatisch als auch lexikalisch differenzierte Formulierungsmuster, die den Autoren erlauben, mit diesen „Textorganisatoren“ flexibel umzugehen, d. h. sie an unterschiedlichen „Gelenkstellen der Argumentation“" (Engel 1988: 105) einzusetzen und mit ihrer Hilfe verschiedene textuelle Funktionen zu realisieren.

\section{d) Präsens vs. Futur I}

Die Vorliebe wissenschaftlicher Texte für das aktuelle Präsens ist allgemein bekannt. Mit seiner Hilfe werden aktuelle, allgemeingültige Untersuchungsergebnisse referiert. Eine niedrigere Frequenz weisen die Tempora Präteritum und Perfekt auf, obwohl sie bei der Beschreibung von durchgeführten Experimenten ebenfalls Anwendung finden. Auch auf der Metaebene wissenschaftlicher Texte sind die genannten Tempora präsent. Während bei Gesamtstrukturierungen, die sich am Ende von Einleitungen befinden und von kumulativ eingebundenen Themaankündigungen konstituiert werden, das Präsens dominant ist, werden in Zusammenfassungen, in denen über Forschungsresultate berichtet wird, Präteritum und Perfekt gebraucht. Das in Themaankündigungen verwendete Präsens ist ein futurisches Präsens. Mit seiner Hilfe informieren die Autoren über die Textplanung, d. h. darüber, welche Themen und Probleme in kommenden Kapiteln zu behandeln sind. Auch neue Kapitel werden häufig durch Themaankündigungen bzw. Zielangaben eröffnet. Das Präsens in Metaformulierungen dieser Art konkurriert nicht selten mit dem Futur I in seiner temporalen, zukunftsorientierten Funktion. Beide Formen gelten als gleichwertige syntaktische Strukturen und werden entweder am Anfang eines neuen oder am Ende eines vorangegangenen Kapitels situiert. Für wissenschaftliche Texte sind sie typische Gliederungssignale, genauer gesagt: Initialsignale oder Vorschaltungen. Beispiele:

Präsens

Im Folgenden wird der Frage nachgegangen, wie ...

In diesem Kapitel sollen ... dargestellt werden.

In diesem Kapitel versuche ich, die Frage zu diskutieren, ...

Im nächsten Kapitel will ich auf ... näher eingehen.
Futur I

Im Folgenden werde ich der Frage nachgehen, wie

In diesem Kapitel werde ich ... darstellen.

Im Folgenden werde ich versuchen, ...

zu eruieren.

Im nächsten Kapitel werde ich auf ...eingehen.

Eine ähnliche grammatische Synonymie lässt sich auch bei Suspensionen, d. h. bei Themaverschiebungen, beobachten. Mit ihrer Hilfe informiert der Autor darüber, dass er ein angesprochenes bzw. kurz behandeltes Problem an einer späteren Textstelle ausführlich(er) 
behandeln wird. Das Präsens scheint bei diesen Metaformulierungen häufiger zu sein. Die Sätze im Futur I gehören jedoch nicht zu „ungewöhnlichen Formen." Beispiele:

Indikativ

Darauf wird in Kap. $\mathrm{x}$ näher eingegangen.

Darauf soll in Kap. $\mathrm{x}$ näher eingegangen werden.

Kapitel $x$ geht auf diese Frage näher ein.

Darauf komme ich in x zurück.

Die Frage ... wird in x näher betrachtet.

Diese Problematik wird in ... noch hinterfragt.
Futur I

Darauf werde ich in Kap. x näher eingehen.

Darauf wird in Kap. $x$ näher einzugehen sein.

Kapitel $\mathrm{x}$ wird auf diese Frage näher eingehen.

Darauf werde ich in ... zurückkommen.

Die Frage ... werde ich in x genauer betrachten.

In Kap. $\mathrm{x}$ wird diese Problematik noch hinterfragt werden müssen.

Vom Autor hängt ab, welche Form er zur Verdeutlichung seiner Absicht wählt. Die Äquivalenz von Präsens und Futur I in den genannten Fällen bildet - so wie in allen oben präsentierten Konkurrenzformen - eine Grundlage für die individuelle Varianz.

\section{e) Satz vs. Ellipse}

Neben Sätzen, die prototypische grammatische Formen und grundlegende Sinneinheiten von schriftlichen Texten darstellen, werden in wissenschaftlichen Texten auch Ellipsen verwendet. Sie lassen sich im Bereich der Mediostrukturen beobachten, d. h. auf der Ebene kleinerer Propositionskomplexe. Nämlich dort, wo die Kommunikationsbedingungen insoweit gesichert sind, dass bestimmte Satzelemente mit routinierten, sich regelmäßig wiederholenden Metaformulierungen eingespart werden können (vgl. Engel 1988: 34, 105). An der Oberfläche bleiben nur Kernbedeutungsträger als Repräsentanten ganzer Äußerungen. Ellipsen nähern den wissenschaftlichen Schriftstil einem Sprechstil an, der z.B. für einen wissenschaftlichen Vortrag charakteristisch ist und es den Autoren erlaubt, sich im Textinneren, d. h. auf der Ebene der Mediostrukturen, weniger formell zu verhalten.

Von Ellipsen machen die Autoren am häufigsten Gebrauch, um von einem Argumentationsschritt zu einem anderen überzugehen. Im Rahmen der gliedernden Funktion handelt es sich hier sowohl um Initialsignale, anders gesagt um sog. „Vorschaltungen“ (Engel 1988: 105), als auch um Wechselsignale. Die Rolle der Initialsignale erfüllen Teilthematisierungen. Nachdem der Autor mehrere Fragen angekündigt hat, beginnt er, diese der Reihe nach zu behandeln. Konkurrieren können in solchen Fällen die folgenden Formen:

Satz

Zunächst soll Punkt 1 näher betrachtet werden.

\section{Ellipse}

Zunächst zum Punkt 1. / Zur ersten Frage: 
Als Initialsignale werden bei der Eröffnung einer neuen thematischen Linie auch elliptische Formen von Rückverweisen in Form von Partizipialkonstruktionen eingesetzt, sei es auf der Ebene von Makrostrukturen, z.B. am Anfang eines neuen Hauptkapitels, sei es auf der Ebene von Mediostrukturen, z.B. am Anfang eines Absatzes:

Als Wechselsignale sind zunächst Positionsmarkierungen zu nennen, die einen Übergang zu einem neuen thematischen Aspekt explizit kennzeichnen, der eine nächste Position in einem Argumentationsstrang einnimmt. Typisch ist dabei die ambikonnexe Partikel nun, die das Neue mit dem gerade Gesagten in Verbindung setzt.

Am Ende einer Ausführung haben die Autoren oft das Bedürfnis, das Gesagte zu ergänzen und sich zu einem Problem zumindest kurz zu äußern, was auch durch entsprechende Metaformulierungen signalisiert wird. Beispiele:

Satz

Nun gehe ich zum Punkt 2 über.

Nun wende ich mich der Frage der ... zu.

$\mathrm{Zu}$... sei noch Folgendes bemerkt: ...
Ellipse

Nun zum Punkt 2:

Nun zur Frage der ... / Nun zur zweiten

Differenz:

Noch ein Wort zu ... / Noch eine Bemerkung zu...

Abschließend sei noch auf Folgendes hingewiesen: ... $\quad$ Abschließend noch eine Bemerkung zu ...

Auch bei der Einbindung neuer Beispiele in den Text finden sich unterschiedliche Formen als Wechselsignale, darunter sowohl satzförmige Konstruktionen als auch Ellipsen, z.B.:

Satz

Noch ein Beispiel ist hier zu nennen.

Ein anderes Beispiel sei hier genannt.

Zur Veranschaulichung sollen folgende Beispiele angegeben werden:

Zur Verdeutlichung will ich folgendes Beispiel nennen: ...

\section{Ellipse}

Noch ein Beispiel: ... Ein anderes Beispiel: ...

Zur Veranschaulichung: ...

Zur Verdeutlichung: ...

Eine Konkurrenz zwischen vollständigen Sätzen und ihren elliptischen Äquivalenten ist auch bei der Ankündigung zusammenfassender Gedanken zum Abschluss einer Argumentation zu beobachten. Typische Metaformulierungen, die einen letzten Absatz eröffnen, sind hier:

Satz

Zusammenfassend ist festzuhalten, dass ...

Zusammenfassend kann festgestellt werden, dass ...
Ellipse

Zusammenfassend: ... Zusammengefasst: ... 
Zusammenfassend muss konstatiert werden, dass ... Als Fazit lässt sich sagen, dass ...

Resümierend ist zu konstatieren, dass ...

Insgesamt lässt sich festhalten, dass ...
Zusammenfassung: ...

Fazit: ... / Zwischenbilanz: ...

Resümee: ... / Resümierend: ...

Insgesamt: ...

Ellipsen erscheinen auch bei Metaformulierungen, die nicht so sehr eine gliedernde, als viel mehr eine verknüpfende Funktion erfüllen. In ihre propositionale Struktur setzen die Autoren konnektive Ausdrücke ein, die explizit auf semantische Kompatibilität der neu eingeführten Sachverhalte hindeuten. Dies lässt sich z.B. bei metatextuell markierten Assertionen, d. h. in metatextuellen Einleitungsformeln solcher Assertionen beobachten, die auf ihre Hauptbedeutungsträger reduziert werden können. Als Hauptbedeutungsträger gelten linkskonnexe Partikeln, die Engel auch „Textorganisatoren“ nennt (1988: 89), und die als Indikatoren für Illokutionen gelten. Die Partikeln somit und also indizieren Konklusionen, und die Partikel immerhin bezeichnet eine Einschränkung. Beide Handlungsmuster, das Schlussfolgern und das Einschränken, sind für wissenschaftliche Texte äußerst charakteristisch. Die folgenden Beispiele wurden einer absatzinitialen Position eines bilanzierenden Absatzes entnommen:

Satz

Somit kann festgestellt werden, dass ...

Es bleibt also festzuhalten, dass ...

Immerhin kann gesagt werden: ...
Ellipse

Somit: ...

Also: ...

Immerhin: ...

Eine konnektive Funktion erfüllen auch die Ellipsen Dazu ein Beispiel oder Dazu folgende Beispiele, die es erlauben, bestimmte Belege zur Bestätigung des früher Gesagten einzubinden und ganze routinierte Sätze einzusparen, etwa in der Form Dazu sollen / können folgende Beispiele genannt werden.

\section{Zusammenfassung}

Ziel des Beitrags war es, die syntaktische Varianz in deutschen wissenschaftlichen Texten aufzuzeigen. Die Varianz lässt sich auf der Metaebene beobachten, d. h. bei der Realisierung von textorganisierenden Handlungen. Berücksichtigt man diese besonders für geisteswissenschaftliche Texte charakteristische Ebene, so wird das syntaktische Bild wissenschaftlicher Fachtexte differenzierter als es gemeinhin angenommen wird. Passivsätze, die für diese Ebene - so wie für die Fachtexte generell - recht typisch sind und einen formellen Stil repräsentieren, konkurrieren mit Aktivsätzen, die eine explizite Verfasserreferenz in Form des Pronomens ich enthalten und eine 'Sprache der Nähe' kennzeichnen. Die kommunikative Funktion der ich-bezogenen Aktivsätze besteht in der Bevorzugung von Direktheit, Natürlichkeit, Authentizität sowie in der Verdeutlichung der eigenen Verantwortung für theoretisch-methodologische Entscheidungen des Autors. 
Aussagesätzen stehen als grammatische Alternativen Imperativsätze gegenüber, die auch die ,Sprache der Nähe' in schriftliche Texte mit sich bringen und den Leser explizit in mentale Operationen und gemeinsame Handlungen einbeziehen. Damit tragen sie zu einer Stilbelebung bei. Neben dem Indikativ als Standardmodus erfreut sich auch der Konjunktiv I in Form von sei + Part. II großer Beliebtheit, da er es ermöglicht, wichtige Inhalte auf kurze Art und Weise zu vermitteln und in unterschiedliche Argumentationsphasen einzubinden. Das Tempus Präsens hingegen hat das zukunftsbezogene Futur I als Äquivalenzform bei der Realisierung von Handlungen, die mit der Planung und Verdeutlichung der Absichten des Autors verbunden sind. Für den Textbildungsprozess ist dies äußerst charakteristisch. Zur syntaktischen Varianz trägt schließlich die Konkurrenz zwischen grammatisch vollständigen Sätzen und ihnen entsprechenden Ellipsen bei. Bestimmte Metaformulierungen werden nämlich auf Kurzformen reduziert, in denen offensichtliche Komponenten regelmäßig wiederkehrender Textroutinen ausgelassen werden können.

Insgesamt weist die syntaktische Metaebene wissenschaftlicher Texte ein großes Potenzial auf. Sie bietet den Autoren ein differenziertes Repertoire von gleichwertigen Strukturen, zwischen denen sie wählen und ihre Texte variieren können, auch wenn die betreffende Textsorte stark durch Routine geprägt ist.

\section{Literatur}

Baumann, Klaus-Dieter (1987): Die Makrostruktur von Fachtexten - ein Untersuchungsansatz. In: Special Language / Fachsprache 1987, 2-18.

Beneš, Eduard (1981): Die formale Struktur der wissenschaftlichen Fachsprachen in syntaktischer Hinsicht. In: Bungarten, Theo (Hg.): Wissenschaftssprache. Beiträge zur Methodologie, theoretischen Fundierung und Deskription. München: Fink, 185-212.

Bungarten, Theo (Hg.) (1981): Wissenschaftssprache. Beiträge zur Methodologie, theoretischen Fundierung und Deskription. München: Fink, 185-212.

Duden-Grammatik (2006): Die Grammatik. Bd. 4, hrsg. von der Dudenredaktion. Mannheim u.a.: Duden.

Ehlich, Konrad (2006): Mehrsprachigkeit in der Wissenschaftskommunikation - Illusion oder Notwendigkeit? In: Ehlich, Konrad / Heller, Dorothee (Hg.): Die Wissenschaft und ihre Sprachen. Frankfurt/M. u.a.: Peter Lang, 17-38.

Engel, Ulrich(1988): Deutsche Grammatik. Heidelberg: Groos.

Engel, Ulrich u.a. (1999): Deutsch-polnische kontrastive Grammatik. Heidelberg: Groos.

Fijas, Liane (1998): Das Postulat der Ökonomie für den Fachsprachengebrauch. In: Hoffmann, Lothar / Kalverkämper, Hartwig / Wiegand, Herbert-Ernst (Hg.): Fachsprachen - Ein internationales Handbuch zur Fachsprachenforschung und Terminologiewissenschaft. 1. Halbband, Berlin u.a.: de Gruyter, 390-97.

Helbig, Gerhard / Buscha, Joachim (2001): Deutsche Grammatik. Ein Handbuch für den Ausländerunterricht. Berlin u.a.: Langenscheidt KG.

Hoffmann Lothar (1984): Kommunikationsmittel Fachsprache. Eine Einführung. Berlin: AkademieVerlag. 
Knobloch, Clemens (2017): Nähe und Distanz - betrachtet aus fachlicher Nähe und aus historiographischer Distanz. In: Feilke, Helmuth / Hennig, Mathilde: Zur Karriere von ,Nähe und Distanz.' Rezeption und Diskussion des Koch-Oesterreicher-Modells. Berlin: de Gruyter, 73-87.

Koch, Peter / Oesterreicher, Wulf (1986): Sprache der Nähe - Sprache der Distanz. Mündlichkeit und Schrifflichkeit im Spannungsfeld von Sprachtheorie und Sprachgeschichte. In: Romanistisches Jabrbuch, Band 36, Berlin: de Gruyter, 15-43.

Kretzenbacher, Heinz L. (1991): Syntax des wissenschaftlichen Fachtextes. In: Fachsprache - Internationale Zeitschrift für Fachsprachenforschung, -didaktik und Terminologie. 13. Jahrgang, Heft 3-4, 118-133.

Kretzenbacher, Heinz L. (1994): Wie durchsichtig ist die Sprache der Wissenschaften? In: Kretzenbacher, Heinz L. / Weinrich, Harald (Hg.): Linguistik der Wissenschaftssprache. Berlin: de Gruyter, $15-40$.

Oksaar, Els (1998): Das Postulat der Anonymität für den Fachsprachengebrauch. In: Hoffmann, Lothar / Kalverkämper, Hartwig / Wiegand, Herbert-Ernst (Hg.): Fachsprachen - Ein internationales Handbuch zur Fachsprachenforschung und Terminologiewissenschaft. 1. Halbband, Berlin u.a.: de Gruyter, 397-401.

Olszewska, Danuta (2007): Metatexteme in den Geisteswissenschaften. Typologie - Funktionalität - Stilistik. Gdańsk: Wydawnictwo Uniwersytetu Gdańskiego.

Olszewska,Danuta (2018 a): Wann sagt ein Wissenschaftler ich? Wann meidet ein Wissenschaftler ich? Über stilistische Tendenzen in Texten junger Wissenschaftler. In: Pittner, Karin / Cirko, Lesław (Hg.): Wissenschaftliches Schreiben interkulturell: Kontrastive Perspektiven. Frankfurt/M.: Peter Lang (im Erscheinen).

Olszewska, Danuta (2018 b): Parlando in wissenschaftlichen Texten. In: Adamzik, Kirsten / Petkova-Kessanlis, Mikaela: Stilwechsel und ihre Funktionen in Textsorten der Fach-und Wissenschaftskommunikation (im Erscheinen).

Panther, Klaus-Uwe (1981): Einige typische indirekte sprachliche Handlungen im wissenschaftlichen Diskurs. In: Bungarten, Theo (Hg.): Wissenschaftssprache. Beiträge zur Methodologie, theoretischen Fundierung und Deskription. München: Fink, 231-260.

Petkova-Kessanlis, Mikaela (2009): Musterhaftigkeit und Varianz in linguistischen Zeitschriftenaufsätzen. Sprachbandlungs-, Formulierungs-, Stilmuster und ihre Realisierung in zwei Teiltexten. Frankfurt/M. u.a.: Peter Lang.

Weinrich Harald (1989): Formen der Wissenschaftssprache. In: Jahrbuch 1988 der Akademie der Wissenschaften zu Berlin. Berlin: de Gruyter, 119-158.

Weinrich, Harald (1993): Textgrammatik der deutschen Sprache. Mannheim u.a.: Dudenverlag. 